\title{
Frequency Domain Analysis of Signals with Stochastic Sampling Times
}

Frida Eng, Fredrik Gustafsson, Fredrik Gunnarsson

Division of Automatic Control

E-mail: frida@isy.liu.se, fredrik@isy.liu.se, fred@isy.liu.se

16th February 2007

Report no.: LiTH-ISY-R-2772

Submitted to IEEE Transactions on Signal Processing.

Address:

Department of Electrical Engineering

Linköpings universitet

SE-581 83 Linköping, Sweden

WWW: http://wwW. control.isy.liu.se

AUTOMATIC CONTROL

REGLERTEKNIK

LINKÖPINGS UNIVERSITET

Technical reports from the Automatic Control group in Linköping are available from http://www. control.isy.liu.se/publications. 


\begin{abstract}
In non-uniform sampling (NUS), signal amplitudes and time stamps are delivered in pairs. Several methods to compute an approximate Fourier transform (AFT) have appeared in literature, and their posterior properties in terms of alias suppression and leakage have been addressed. In this paper, the sampling times are assumed to be generated by a stochastic process. The main result gives the prior distribution of several AFTs expressed in terms of the true Fourier transform and variants of the characteristic function of the sampling time distribution. The result extends leakage and alias suppression with bias and variance terms due to NUS. Specific sampling processes as described in literature are analyzed in detail. The results are illustrated on simulated signals, with particular focus to the implications for spectral estimation.
\end{abstract}

Keywords: frequency domain, stochastic sampling, signal processing 


\title{
Frequency Domain Analysis of Signals with Stochastic Sampling Times
}

\author{
Frida Eng, Fredrik Gustafsson and Fredrik Gunnarsson
}

$2007-12-16$

\begin{abstract}
In non-uniform sampling (NUS), signal amplitudes and time stamps are delivered in pairs. Several methods to compute an approximate Fourier transform (AFT) have appeared in literature, and their posterior properties in terms of alias suppression and leakage have been addressed. In this paper, the sampling times are assumed to be generated by a stochastic process. The main result gives the prior distribution of several AFTs expressed in terms of the true Fourier transform and variants of the characteristic function of the sampling time distribution. The result extends leakage and alias suppression with bias and variance terms due to NUS. Specific sampling processes as described in literature are analyzed in detail. The results are illustrated on simulated signals, with particular focus to the implications for spectral estimation.
\end{abstract}

\section{Introduction}

Non-uniform sampling (NUS) occurs in many applications where uniform sampling is either not possible or practically not achievable:

Event based sampling in the time domain In queue processes, the signal will change level for each arrival and departure, which occur naturally on a non-uniform time grid. One example is routers in data networks, where data packets come and go and the queue length is a central control parameter. Here, it would not make sense to sample the queue length uniformly. Another example is analysis of astronomical data, where for instance the signal may be the number of received neutrinos. Missing data is another kind of event process, where the sampling times can be modeled as stochastic.

Uniform sampling in other domains Angular speeds are often computed from regular angular displacement. One example is angular speed sensors, e.g., ABS in cars, that give a certain number of pulses for each wheel revolution. The samples are uniform in the angle domain, but non-uniform in the time domain. Another example is the heart rate spectrum, which is computed from the peak times of an ECG signal. Again, the sampling is uniform in the heart rate domain, but non-uniform in the time domain.

User chosen non-uniform grid In some applications, a non-uniform time grid is desirable, e.g., to avoid detection in military radar applications. 
Also the time grid can be chosen to avoid aliasing or increase other performance parameters.

In all cases, a posteriori analysis can be based on interpolating the signal to a uniform time grid and use classical Fourier transform (FT) based approaches to frequency, spectral or model-based analysis. The interpolation error is in Schoukens et al. (1992) shown to be quite small if the interpolation is done correctly. There are also several methods to compute an approximate FT (AFT) (Beutler, 1970; Benedetto, 1992; Feichtinger and Gröchenig, 1994; Beurling, 1989).

Posterior analysis of AFTs in terms of alias suppression and leakage is a fairly well explored area (Lomb, 1976; Scargle, 1982; Wojtkiewicz and Tuszyński, 1992). The reverse problem of designing NUS to maximize alias suppression is also well studied (Papenfuss et al., 2003; Bland and Tarczynski, 1997; Shapiro and Silverman, 1960; Masry, 1978, 1983; Hall and Yin, 2004).

This article addresses the problem of a priori analysis of the stochastic properties of certain natural Fourier transform approximations. More specifically, we derive the asymptotic distribution for the approximate Fourier transform (AFT) in terms of the true signal's Fourier transform, and a stochastic model of the sampling times, before the pairwise measurements of the signal and time are performed. This is done for some important cases of sampling time noise. In this way, one can compute an a priori confidence region for each frequency in the AFT. Once the signal and time stamps are observed, we get one observation of the AFT (from the distribution we have derived), that will lie in this confidence region with a certain probability.

Focus is on frequency resolution, alias suppression and stochastic uncertainty in the AFT. Given the results, it is possible to evaluate intricate design questions for the applications mentioned above, and some examples include: How well can the eigen-frequencies of a car tire be determined, given that the sampling times are perturbed by the car speed and road roughness? Is it possible to find the oscillations in a router in the Internet, to make improvements of its control protocols possible? Given that neutrinos arrive according to a Poisson process, how many arrivals are needed to find the dominating frequencies with a certain accuracy? How does the outlier probability influence the bias in spectral estimates?

In Section 2, the signal model and stochastic frequency model are defined, and different non-uniform sampling schemes are described. A discussion of interpolation techniques for approximating the true transform is done in Section 3. The main part of the work is the analysis of the stochastic properties of two linear frequency transforms, with the main results in Section 4, together with extensions for the periodogram. In Section 5, asymptotic analysis of the mean value and the distribution is done, which further increase the knowledge about the AFT and the periodogram. Finally, Section 6 gives examples of both the AFT and the periodogram. The article is concluded in Section 7.

\section{Problem Definition}

We start by describing the signal model, and clarifying the notation. This leads to a description of the stochastic frequency model. Different stochastic sampling models are listed at the end. The notation adopted in this work is summarized 
Table 1: Notation and relevant references.

\begin{tabular}{rll}
$s(t)$ & continuous-time signal & $(1)$ \\
$S(f)$ & true Fourier transform of signal $s(t)$ & $(1)$ \\
\hline$t_{k}$ & non-uniform stochastic sampling time & $(3)$ \\
$p_{k}(t)$ & probability density function $(\mathrm{pdf})$ for $t_{k}$ & $(2)$ \\
$\tau_{k}$ & sampling noise, for example, $t_{k}=k T+\tau_{k}$ & Section 2.3 \\
$p_{\tau}(t)$ & pdf for the sampling noise, $\tau$ & \\
$\varphi_{\tau}(f)$ & characteristic function for $\tau$ & Section 4 \\
$T_{k}$ & inter-sampling times, $t_{k}-t_{k-1}, t_{0}=0$ & \\
\hline$y_{k}$ & stochastic sample of $s(t)$ at time $t_{k}$ & $(3)$ \\
$w_{k}(\cdot)$ & stochastic frequency weight & $(4) \&(19)$ \\
$Y(\cdot)$ & stochastic frequency transform of $y_{k}$ & $(7)$ \\
$W(\cdot)$ & stochastic frequency window & $(25)$ \\
\hline$a_{1}^{n}$ & the sequence $a_{k}, k=1, . ., n$. & \\
$g(\cdot \mid t)$ & deterministic value of the stochastic func- & \\
& tion $g(\cdot)$ conditioned on $t$ \\
$f(\cdot ; \theta)$ & the function $f(\cdot)$ has implicit dependence &
\end{tabular}

in Table 1, together with relevant references to descriptions or definitions in the text.

\subsection{Signal Model}

We consider a deterministic continuous-time signal, $s(t)$, with Fourier transform $S(f)$

$$
s(t)=\int_{-\infty}^{\infty} S(f) e^{i 2 \pi f t} d f
$$

which is non-uniformly sampled $N$ times. The sample times, $t_{k}$, are stochastic variables with probability density functions (pdfs),

$$
p_{k}(t)=\left\{\begin{aligned}
\frac{d}{d t} P\left(t_{k} \leq t\right) & \text { continuous density } \\
P\left(t_{k}=t\right) & \text { discrete density }
\end{aligned}\right.
$$

for $k=1, . ., N$, and the number of samples, $N$, is deterministic. In the following, we consider only continuous density functions, $p_{k}(t)$. From the continuous-time signal (or function) $s(t)$ we get a stochastic observation, $y_{k}$, of the corresponding deterministic sample value:

$$
y_{k}=s\left(t_{k}\right)
$$

Thus, $y_{k}$ is a function of the stochastic variable $t_{k}$ and its stochastic properties can be investigated accordingly. For example, its mean is

$$
\mathrm{E}\left[y_{k}\right]=\mathrm{E}\left[s\left(t_{k}\right)\right]=\int_{-\infty}^{\infty} s(t) p_{k}(t) d t .
$$

Any transform of these stochastic function values or measurements, $y_{k}$, can be investigated to study its a priori properties, for example, for parameter estimation or frequency analysis. In this work, we consider the case of frequency 
analysis of a continuous-time signal, $s(t)$, sampled non-uniformly and then transformed to approximate the true Fourier transform, $S(f)$.

Sampling of stochastic functions will not be considered here. However, it will be clear that additive measurement noise easily can be included in the descriptions, since only linear transformations of the measurements are of interest. By adding zero-mean measurement noise, $e_{k}$, to the signal sample we, for example, get $\mathrm{E}\left[s\left(t_{k}\right)+e_{k}\right]=E\left[s\left(t_{k}\right)\right]$.

\subsection{Stochastic Frequency Model}

The transforms of interest in this work are linear transforms of the measurements, that can be calculated when the measurement times are given, i.e.,

$$
Y\left(f \mid t_{1}^{N}\right)=\sum_{k=1}^{N} y_{k} w_{k}\left(f \mid t_{k}\right)
$$

where $Y\left(f \mid t_{1}^{N}\right)$ is the deterministic approximation, AFT, of the true transform, $S(f)$. Here, $w_{k}\left(f \mid t_{1}^{N}\right)$ are weights to be decided, see the coming discussion in Section 3 , and $t_{1}^{N}$ denotes the sequence of the sampling times,

$$
t_{1}^{N}=\left(t_{k}\right)_{k=1}^{N} .
$$

For uniform sampling, $t_{k}=k T$, the discrete time Fourier transform (DTFT) is commonly used

$$
Y_{\mathrm{dtft}}(f)=T \sum_{k=1}^{N} s(k T) e^{-i 2 \pi f k T}
$$

i.e., it corresponds to weighting with $w_{k}(f \mid k T)=T e^{-i 2 \pi f k T}$. The literature contains definitions both with and without the scaling factor $T$. The well-known discrete Fourier transform (DFT) is the DTFT evaluated at discrete frequency points, normally without the scaling.

Using Bayes' rule we can derive the pdf for the random variable $Y(f)$, denoted $p_{Y(f)}(y)$, by marginalization of the stochastic sampling times,

$$
p_{Y(f)}(y)=\int_{-\infty}^{\infty} p_{Y\left(f \mid t_{1}^{N}\right)}(y) p\left(t_{1}^{N}\right) d t_{1}^{N},
$$

where the joint pdf of the sampling times, $p\left(t_{1}^{N}\right)$, will depend on the type of sampling process. We also note that, since $Y\left(f \mid t_{1}^{N}\right)$ is deterministic, $p_{Y\left(f \mid t_{1}^{N}\right)}(y)=$ $\delta\left(y-Y\left(f \mid t_{1}^{N}\right)\right)$. This gives, with (7), the a priori distribution of $Y(f)$,

$$
p_{Y(f)}(y)=\int \delta\left(y-Y\left(f \mid t_{1}^{N}\right)\right) p\left(t_{1}^{N}\right) d t_{1}^{N},
$$

which would require a change of variables in order to be solved. The a priori distribution of $Y(f)$ enables investigation of, for example, the mean value and covariance achieved for different sampling processes, expected leakage and aliasing. A quick investigation shows that this expression is mainly for theoretical discussions, since it is virtually impossible to evaluate, so we will use other means to explicitly derive mean value and covariance for the stochastic AFT. 


\section{$2.3 \quad$ Non-Uniform Sampling}

Non-uniform sampling can occur in different forms and this section lists the most common sampling models. The probability distribution for the sampling times, $t_{k}$, was given as $p_{k}(t)$ in (2). Depending on the type of sampling, $p_{k}(t)$ can be deduced from the probability density function, $p_{\tau}(t)$ of the sampling noise, $\tau_{k}$.

For additive random sampling (ARS), the sampling times are constructed by adding the sampling noise to the previous sampling time,

$$
t_{k}=t_{k-1}+\tau_{k}=\sum_{n=1}^{k} \tau_{n}, \quad t_{0}=0
$$

where $\tau_{k} \in(0, \infty)$ are i.i.d. stochastic noise and $\mathrm{E}\left[\tau_{k}\right]=T$. This means that $\mathrm{E}\left[t_{k}\right]=k T$, while the variance increases with $k$. The pdf is given as a convolution of the sampling noise pdf $k$ times,

$$
p_{k}(t)=\underbrace{p_{\tau} \star \ldots \star p_{\tau}}_{k \text { times }}(t) \triangleq p_{\tau}^{(k)}(t) .
$$

For example, the exponential distribution, $p_{\tau}(t)=1 / T e^{-t / T}$, gives a Poisson sampling process. The central limit theorem gives that $p_{k}(t)$ will approach a Gaussian distribution when $k$ goes to infinity, since it is the pdf of a sum of $k$ independent identically distributed variables (9).

For stochastic jitter sampling (SJS), the sampling noise is added to the expected sampling time,

$$
t_{k}=k T+\tau_{k}
$$

with $\tau_{k} \in(-T / 2, T / 2)$ being i.i.d. stochastic noise and $\mathrm{E}\left[\tau_{k}\right]=0$. In this case the variance is constant over time and the pdf is given directly by the pdf for $\tau$,

$$
p_{k}(t)=p_{\tau}(t-k T)
$$

One natural distribution is the rectangular distribution,

$$
p_{\tau}(t)=1 / T, \quad t \in[-T / 2, T / 2],
$$

or more generally the beta distribution

$$
\begin{array}{rlrl}
p_{\tau}(t) & =\frac{1}{\mathrm{~B}(\alpha, \beta)} t^{\alpha-1}(1-t)^{\beta-1}, & & t \in[0,1] \\
\mathrm{B}(\alpha, \beta) & =\frac{\Gamma(\alpha) \Gamma(\beta)}{\Gamma(\alpha+\beta)}, & \alpha, \beta>0,
\end{array}
$$

where $\Gamma(q)=\int t^{q-1} e^{-t} d t$ is the gamma-function ${ }^{1}$. The beta distribution is the rectangular distribution when $\alpha=\beta=1$.

Another special case is the problem with missing data (MD), where the underlying sampling procedure is uniform but sometimes samples are missed. This can, in the current context, be described with a discrete sampling noise,

$$
t_{k}=t_{k-1}+\tau_{k}
$$

\footnotetext{
${ }^{1} \Gamma(m)=(m-1)$ ! for integer values, $m>0$.
} 
and $\tau_{k} \in\{T, 2 T, \ldots\}$. This can be seen as a special case of ARS, with a discrete pdf for the sampling noise, for example, $p_{\tau}(n T)=P\left(\tau_{k}=n T\right)=p(1-p)^{n-1}$ gives a "First success" distribution. The expected value $\mathrm{E}\left[t_{k}\right]>k T$ whenever $\mathrm{E}\left[\tau_{k}\right]>T$, which is the case for every nontrivial pdf. The missing data problem is also discussed in (Ljung, 1999, Ch. 14) with further references. In Table 2, these sampling types are summarized with mean value of the sampling times, support of the sampling noise and the update equation for the sampling times and the pdf.

Table 2: Summary of different sampling models, additive random sampling (ARS), stochastic jitter sampling (SJS) and missing data (MD).

\begin{tabular}{|l|l|c|c|c|}
\hline Type & Update & $\mathrm{E}\left[t_{k}\right]$ & $\tau_{k} \in$ & $p_{k}(t)$ \\
\hline ARS & $t_{k}=t_{k-1}+\tau_{k}$ & $k T$ & $(0, \infty)$ & $p_{\tau}^{(k)}(t)$ \\
\hline SJS & $t_{k}=k T+\tau_{k}$ & $k T$ & $(-T / 2, T / 2)$ & $p_{\tau}(t-k T)$ \\
\hline MD & $t_{k}=t_{k-1}+\tau_{k}$ & $>k T$ & $\{T, 2 T, \ldots\}$ & - \\
\hline
\end{tabular}

\section{Approximation of the Fourier Transform}

Non-uniform sampling, both deterministic and stochastic, is described in Bilinskis and Mikelsons (1992) and Marvasti (2001). Among other things, the literature describes how the Fourier transform (FT) can be approximated when the sampling times are deterministic.

\subsection{Two Transform Expressions}

As stated earlier, we will investigate the use of linear transforms of the form given in (4). Our interest is focused on approximating the continuous-time Fourier transform

$$
S(f)=\int_{-\infty}^{\infty} s(t) e^{-i 2 \pi f t} d t=\int_{-\infty}^{\infty} I(t) d t,
$$

with such a transformation, $Y\left(f \mid t_{1}^{N}\right)$, of the discrete-time measurements. These approximations are done based on knowledge of the sample times, $t_{k}$, and actual measurements, $y_{k}=s\left(t_{k}\right)$, and can then be used to design the transform, via (7), of the stochastic sample values that we aim for.

Using zero order spline interpolation of the integrand $I(t)$, corresponds to Riemann integration and yields

$$
Y\left(f \mid t_{1}^{N}\right)=\sum_{k=1}^{N} T_{k} y_{k} e^{-i 2 \pi f t_{k}}
$$

called the Riemann transform (RieT).

In several contexts, the Dirichlet transform (DirT) has been proposed as a suitable frequency transform for non-uniformly sampled signals (Bilinskis and Mikelsons, 1992; Tarczynski and Allay, 2004). It can be deduced, for example, 
by approximating the signal $s(t)$ with an impulse train. The Dirichlet transform is given as

$$
Y\left(f \mid t_{1}^{N}\right)=\sum_{k=1}^{N} y_{k} e^{-i 2 \pi f t_{k}}
$$

Except for the scaling factor $T$, both expressions coincide with the DTFT (6) when $t_{k}=k T$.

\subsection{Related Work}

There are other numerical integration methods, for example, Runge-Kutta integration and Newton-Cotes formulas, that could be used for transforms similar to RieT. In Bland et al. (1996), several integration methods are also investigated for non-uniform sampling. In Persson (2002) and van Steenis and Tulen (1991), the RieT is used in applications.

The DirT can be compared with the Lomb-Scargle periodogram (Lomb, 1976; Scargle, 1982), which is a least squares fitting of the transform at each frequency, and is approximately the $|\operatorname{DirT}|^{2}$, only scaled with $N$. In Gunnarsson et al. (2004), a few more expressions are derived, for example, with spline interpolation and sinc basis expansion (for band limited functions). The expressions are much more complicated to calculate and for almost uniform sampling, they basically coincide with the RieT and DirT.

\subsection{Summarizing the Expressions}

In summary, this work focuses on two AFTs given by (4) with,

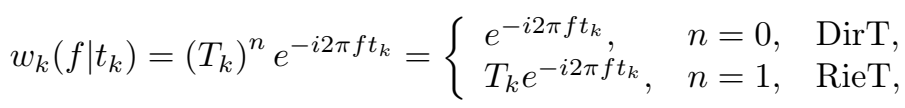

where $n \in\{0,1\}$ indicates the transform type used.

In the remainder of this paper, the sampling times, $t_{k}$, are stochastic variables, and the frequency transform, $Y(f)$, is a random variable with a certain probability distribution. We will investigate the properties of this distribution depending on the type of sampling chosen from Table 2 and the type of transform in (19).

\subsection{The Periodogram}

Here, we define the periodogram $P_{Y}(f)$ of $Y(f)$ as

$$
P_{Y}(f)=|Y(f)|^{2},
$$

and get another stochastic variable. Other definitions of $P_{Y}$ can include scaling with the number of samples $N$, or the total time interval, $t_{N}$ or $N \mu_{T} \mathrm{E}\left[T_{k}\right]$, which does not affect the results.

The periodogram for zero-mean Gaussian signals is known to be exponentially distributed. Stoica and Moses (2005) and Ljung (1999) shows that

$$
\left|\frac{1}{N} \sum v_{k} e^{-i 2 \pi f k T}\right|^{2} \in A s \mathcal{E} \operatorname{xp}\left(1 / \sigma^{2}\right),
$$


when $v_{k}$ is (uniformly sampled) zero-mean white Gaussian noise. We will return to this when discussing the distribution of the periodogram in the case of nonuniform sampling. Note that in the literature, the above periodogram is said to be chi-squared distributed with two degrees of freedom, which is the same as the exponential distribution. We choose the exponential notation to give good correspondance with the following investigation.

\section{Main Results}

The condensed result of this work is given in this section, and it is discussed and exemplified in the following sections.

It is obvious that the distribution of $\tau_{k}$ plays a crucial role for the stochastic properties of the AFT. This will be shown via the influence of the characteristic function, $\varphi_{\tau}(\omega)=E_{\tau}\left[e^{i \omega \tau}\right]$. It will be convenient to define an extended version of the characteristic function

$$
\varphi_{m}(f) \triangleq \mathrm{E}\left[\left(T_{k}\right)^{m} e^{-i 2 \pi f \tau_{k}}\right], \quad m=0,1, \ldots
$$

where $T_{k}$ is the inter-sampling time and $m=0$ gives the standard definition when $f=-\omega / 2 \pi$. The parameter $m$ will show parts of the difference between the two transforms, RieT and DirT. A strong connection with the uniform sampling case will be shown with the appearance of the normalized Dirichlet kernel (also known as the aliased sinc function)

$$
d_{N}^{\mathrm{SJS}}(f) \triangleq e^{-i 2 \pi f T} \frac{1-e^{-i 2 \pi f N T}}{1-e^{-i 2 \pi f T}}=e^{-i \pi f(N-1) T} \frac{\sin (\pi f N T)}{\sin (\pi f T)} .
$$

A more general definition will also occur,

$$
d_{N}^{\mathrm{ARS}}(f) \triangleq \frac{1-\varphi_{0}(f)^{N}}{1-\varphi_{0}(f)}=\frac{1-\mathrm{E}\left[e^{-i 2 \pi f \tau_{k}}\right]^{N}}{1-\mathrm{E}\left[e^{-i 2 \pi f \tau_{k}}\right]} .
$$

The superscripts indicate that these factors will show the difference between the sampling types.

\subsection{Mean and Covariance of $Y(f)$}

In the following we study the a priori properties, and hence the conditioning on $t_{1}^{N}$ is removed. We consider a signal $s(t)$ with Fourier transform $S(f)$ sampled non-uniformly at random times $t_{k}$, with probability densities, $p_{k}(t)$, resulting in random signal samples $y_{k}=s\left(t_{k}\right)$. The sampling times are constructed from random sampling noise, $\tau_{k}$, using one of ARS and SJS, see Table 2. If we use the inverse Fourier transform description, (1), we get

$Y(f)=\sum_{k=1}^{N} y_{k} w_{k}(f)=\sum_{k=1}^{N}\left(\int_{-\infty}^{\infty} S(\psi) e^{i 2 \pi \psi t_{k}} d \psi\right) w_{k}(f)=\int_{-\infty}^{\infty} S(\psi) W(f, \psi) d \psi$

where

$$
W(f, \psi)=\sum_{k=1}^{N} e^{i 2 \pi \psi t_{k}} w_{k}(f)
$$


The definition of the weights, $w_{k}(f)$, in (19), implies that

$$
W(f, \psi)=W(f-\psi) \triangleq \sum_{k=1}^{N}\left(T_{k}\right)^{n} e^{-i 2 \pi(f-\psi) t_{k}},
$$

which gives a nice convolution formulation in (24a), and allows us to state the first two moments of $Y$.

Theorem 1 (Mean Value and Covariance of $Y$ ). The mean value, $\mu_{Y}$, and covariance, $R_{Y}$, of the stochastic frequency transform, $Y(f)(24)$, are

$$
\begin{aligned}
\mu_{Y}\left(f ; S, p_{1}^{N}\right) & =S * \mu_{W}\left(f ; p_{1}^{N}\right), \\
R_{Y}\left(f, \psi ; S, p_{1}^{N}\right) & =S * * R_{W}\left(f, \psi ; p_{1}^{N}\right),{ }^{2}
\end{aligned}
$$

with

$$
\mu_{W}\left(f ; p_{1}^{N}\right) \triangleq E[W(f)]
$$

and

$$
R_{W}\left(f, \psi ; p_{1}^{N}\right) \triangleq \operatorname{Cov}(W(f), W(\psi)) .
$$

Proof. By using (24) we get the mean value of $Y$ as

$$
\begin{aligned}
\mu_{Y}\left(f ; S, p_{1}^{N}\right) & \triangleq \mathrm{E}[Y(f)]=\mathrm{E}\left[\int_{-\infty}^{\infty} S(\phi) W(f-\phi) d \phi\right] \\
& =\int_{-\infty}^{\infty} S(\phi) \mathrm{E}[W(f-\phi)] d \phi=S * \mu_{W}\left(f ; p_{1}^{N}\right) .
\end{aligned}
$$

In a similar manner the covariance is obtained,

$$
\begin{aligned}
R_{Y}\left(f, \psi ; S, p_{1}^{N}\right) \triangleq & \operatorname{Cov}(Y(f), Y(\psi)) \\
= & \mathrm{E}\left[\left(Y(f)-\mu_{Y}(f)\right)\left(Y(\psi)-\mu_{Y}(\psi)\right)^{*}\right] \\
= & \mathrm{E}\left[\left(\int_{-\infty}^{\infty} S(\phi)\left(W(f-\phi)-\mu_{W}(f-\phi)\right) d \phi\right) \times\right. \\
& \left.\times\left(\int_{-\infty}^{\infty} S(\eta)\left(W(\psi-\eta)-\mu_{W}(\psi-\eta)\right) d \eta\right)^{*}\right] \\
= & \iint_{-\infty}^{\infty} S(\phi) \mathrm{E}\left[\left(W(f-\phi)-\mu_{W}(f-\phi)\right) \times\right. \\
& \left.\times\left(W(\psi-\eta)-\mu_{W}(\psi-\eta)\right)^{*}\right] S(\eta)^{*} d \phi d \eta \\
= & S * * R_{W}\left(f, \psi ; p_{1}^{N}\right),
\end{aligned}
$$

and the proof is complete.

The connection to the original sampled signal is completely captured in the convolutions with $S(f)$. This is why we can focus on the stochastic frequency window, $W$, and its properties. For a single sinusoid, the transform is given as $Y(f)=\left(W\left(f-f_{0}\right)-W\left(f+f_{0}\right)\right) / 2 i$, and the stochastic frequency window is the sampling effect on a single frequency. This is completely in accordance with the uniform sampling case.

\footnotetext{
${ }^{2}$ We use $*$ to denote single convolution, $f * g(x)=\int f(\xi) g(x-\xi) d \xi$, and $* *$ for double, $f * * h(x, y)=\iint f(\xi) h(x-\xi, y-\eta) f^{*}(\eta) d \xi d \eta$, respectively.
} 


\subsection{Mean and Covariance of $W(f)$}

As shown in Theorem 1, we can focus on the stochastic frequency window, $W$, to describe the properties of the original transform. The mean value and covariance can be described completely with the following theorem.

Theorem 2 (Mean Value and Covariance of $W$ ). The mean value, $\mu_{W}$, and covariance, $R_{W}$, (defined in (27)) of the stochastic frequency window, $W(\cdot)(25)$, are

$$
\begin{aligned}
\mu_{W}\left(f ; p_{1}^{N}\right) & =\varphi_{n}(f) d_{N}^{S T}(f), \\
R_{W}\left(f, \psi ; p_{1}^{N}\right) & =\Phi_{n}^{S T}(f, \psi)^{T} D_{N}^{S T}(f, \psi)-\mu_{W}(f) \mu_{W}(\psi)^{*},
\end{aligned}
$$

where the explicit expressions are given in Table 3. The sampling type, ST, is one of $\{S J S, A R S\}$ and the transform type affects $n \in\{0,1\}$, giving four combinations, summarized in Table 4.

Proof. When considering ARS, (9), the inter-sampling time is exactly the sampling noise, $T_{k}=\tau_{k}$. The stochastic properties of the frequency window, $W(\cdot)$, will depend on the finite number of samples, $N$, and the stochastic properties of the sampling noise.

The mean value of the stochastic frequency window, for the case of ARS, is

$$
\begin{aligned}
\mu_{W}\left(f ; p_{1}^{N}\right) & =\mathrm{E}[W(f)]=\sum_{k=1}^{N} \mathrm{E}\left[\left(T_{k}\right)^{n} e^{-i 2 \pi f t_{k}}\right] \\
& =\sum_{k=1}^{N} \mathrm{E}\left[\left(T_{k}\right)^{n} \prod_{n=1}^{k} e^{-i 2 \pi f \tau_{n}}\right]=\sum_{k=1}^{N} \mathrm{E}\left[\left(T_{k}\right)^{n} e^{-i 2 \pi f \tau_{k}}\right] \varphi_{0}(f)^{k-1} \\
& =\varphi_{n}(f) \sum_{k=0}^{N-1} \varphi_{0}(f)^{k}=\varphi_{n}(f) d_{N}^{\mathrm{ARS}}(f)
\end{aligned}
$$

which corresponds to (28a) and the definitions in Table 3.

For SJS, (11), the first moment is very similar,

$\mathrm{E}[W(f)]=\sum_{k=1}^{N} e^{-i 2 \pi f k T} \mathrm{E}\left[\left(T_{k}\right)^{n} e^{-i 2 \pi f \tau_{k}}\right]=\varphi_{n}(f) \sum_{k=1}^{N} e^{-i 2 \pi f k T}=\varphi_{n}(f) d_{N}^{\mathrm{SJS}}(f)$,

which also corresponds to the given definitions.

The calculations for the covariance of $W(\cdot)$ are straightforward and similar to the ones above. They are given in Appendices A and B to keep the presentation clean. Note that for SJS and DirT, the expressions are more compact than they appear, since $n=0$ and then the term $n \kappa(f) \varphi_{0}(-\psi)=0$.

For uniform sampling and a uniform (boxcar) time window, the frequency window, $W$, is deterministic and becomes exactly the normalized Dirichlet kernel, $d_{N}^{\mathrm{SJS}}(f)$, and the corresponding transform,

$$
Y(f \mid k T)=\int_{-\infty}^{\infty} S(\psi) d_{N}^{\mathrm{SJS}}(f-\psi) d \psi,
$$


Table 3: Explicit expressions needed for evaluation of Theorem 2. The indicator $n \in\{0,1\}$ specifies the transform.

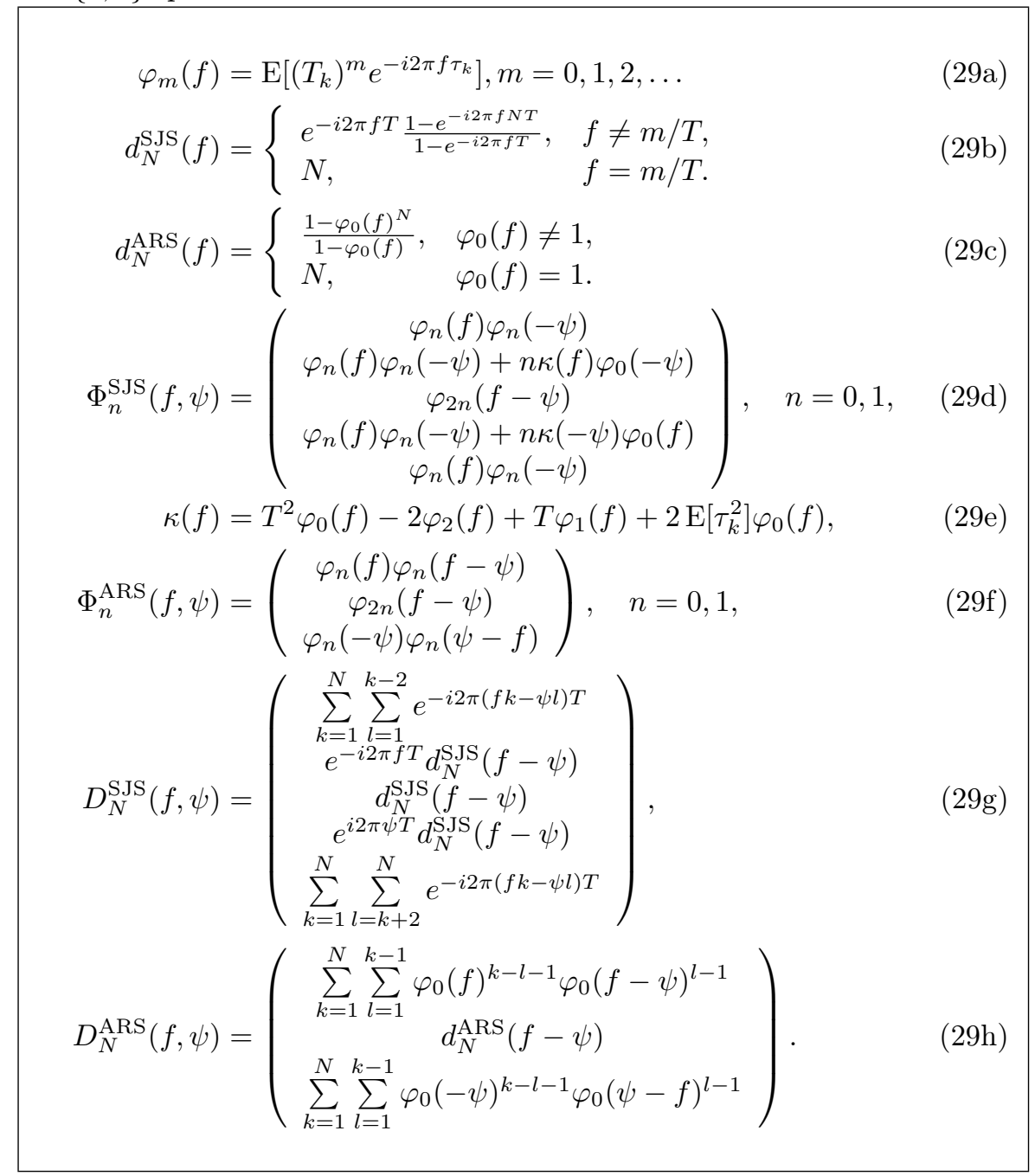

Table 4: Collection of expressions for different setups of (28a) and (28b) in Theorem 2. Table 3 defines the functions.

Theorem 2. Table 3 defines the functions.
\begin{tabular}{|c|c|c|c|}
\hline & & $\operatorname{DirT}(n=0)$ & $\operatorname{RieT}(n=1)$ \\
\hline \multirow{2}{*}{ ARS } & $\mu_{W}$ & $\varphi_{0}(f) d_{N}^{\mathrm{ARS}}(f)$ & $\varphi_{1}(f) d_{N}^{\mathrm{ARS}}(f)$ \\
& $R_{W}+\mu \mu^{*}$ & $\Phi_{0}^{\mathrm{ARS}}(f, \psi)^{T} D_{N}^{\mathrm{ARS}}(f, \psi)$ & $\Phi_{1}^{\mathrm{ARS}}(f, \psi)^{T} D_{N}^{\mathrm{ARS}}(f, \psi)$ \\
\hline \multirow{2}{*}{ SJS } & $\mu_{W}$ & $\varphi_{0}(f) d_{N}^{\mathrm{SSS}}(f)$ & $\varphi_{1}(f) d_{N}^{\mathrm{SJS}}(f)$ \\
& $R_{W}+\mu \mu^{*}$ & $\Phi_{0}^{\mathrm{SJS}}(f, \psi)^{T} D_{N}^{\mathrm{SJS}}(f, \psi)$ & $\Phi_{1}^{\mathrm{SJS}}(f, \psi)^{T} D_{N}^{\mathrm{SJS}}(f, \psi)$ \\
\hline
\end{tabular}


is the DTFT, (6). Previous publications include analysis of the stochastic frequency window, $W(f)$. Studies of the expected value are made in Gunnarsson et al. (2004), and signal estimation in the frequency domain for non-uniformly sampled signals is investigated in Eng and Gustafsson (2005b).

Remark 1. For decreasing sampling noise variance the different sampling types tend to uniform sampling. The results for the mean value agree with this since, intuitively, we have

$$
\begin{aligned}
\varphi_{n}(f) & \rightarrow T^{n}, \quad \text { when } \operatorname{Var}\left(\tau_{k}\right) \rightarrow 0, \mathrm{E}\left[\tau_{k}\right]=0, \\
d_{N}^{A R S}(f) & \rightarrow d_{N}^{S J S}(f), \text { when } \operatorname{Var}\left(\tau_{k}\right) \rightarrow 0, \mathrm{E}\left[\tau_{k}\right]=T,
\end{aligned}
$$

and hence E[DirT] and E[RieT] approach the DTFT.

\subsection{The Periodogram}

With the previous results we can explicitly get the mean of the periodogram.

Corollary 1 (Mean of the Periodogram). The mean value $\mu_{P}(f)$ of the periodogram $P_{Y}(f)(20)$ is

$$
\mu_{P}(f)=\mathrm{E}\left[|Y(f)|^{2}\right]=\left|\mu_{Y}(f)\right|^{2}+R_{Y}(f, f),
$$

where $\mu_{Y}$ and $R_{Y}$ are given by Theorem 1 .

Proof. The result is given by the definition of the variance

$$
R_{Y}(f, f)=\mathrm{E}\left[\left|Y(f)-\mu_{Y}(f)\right|^{2}\right]=E\left[|Y(f)|^{2}\right]-\left|\mu_{Y}(f)\right|^{2}=\mu_{P}(f)-\left|\mu_{Y}(f)\right|^{2} .
$$

\section{Asymptotic Analysis for ARS}

When the number of measurements increases to infinity more conclusions can be made. Here, we give results for the asymptotic mean value, and discuss asymptotic distributions for the transform, its magnitude, and the periodogram.

\subsection{Asymptotic Analysis of the Mean Value}

In the previous analysis, a finite number of samples, $N$, was considered. The following lemma investigates the term $d_{N}^{\mathrm{ARS}}(f)$, when $N$ goes to infinity. The periodic counterpart, $d_{N}^{\text {SJS }}(f)$, is well known and thoroughly studied, it will converge to an impulse train as $N$ increases. The lemma will provide us with a tool to study the asymptotic mean value of $W(f)$ and $Y(f)$ for ARS. Note that, in the lemma, we discuss distributions from functional theory and not probability theory. The assumptions in the lemma will be discussed later.

Lemma 1 (Asymptotic Properties of $d_{N}^{\mathrm{ARS}}$ ). Consider the case of ARS. Assume that the continuous-time function $h(t)$ with FT $H(f)$ fulfills the following conditions:

1. $h(t)$ and $H(f)$ belongs to the Schwartz class, $\mathcal{S} .^{3}$

\footnotetext{
${ }^{3} h \in \mathcal{S} \Leftrightarrow t^{k} h^{(l)}(t)$ is bounded, i.e., $h^{(l)}(t)=\mathcal{O}\left(|t|^{-k}\right)$, for all $k, l \geq 0$.
} 
2. The sum $g_{N}(t)=\sum_{k=1}^{N} p_{k}(t)$ obeys

$$
\lim _{N \rightarrow \infty} \int g_{N}(t) h(t) d t=\int \frac{1}{\mu_{T}} h(t) d t=\frac{1}{\mu_{T}} H(0),
$$

for this $h(t)$.

3. The initial value is zero, $h(0)=0$.

Then, it holds that

$$
\lim _{N \rightarrow \infty} \int d_{N}^{A R S}(f) H(f) d f=\frac{1}{\mu_{T}} H(0) .
$$

Proof. The proof is conducted using distributions. ${ }^{4}$ Let $\Delta_{r}$ denote the distribution corresponding to the function $r$. We use

$$
\left\langle\Delta_{r}, h\right\rangle=\int r(t) h(t) d t
$$

to denote the action of the distribution corresponding to $r$ on $h \in \mathcal{S}$. Now, the aim is to show that

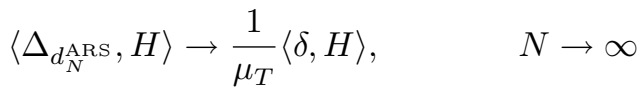

under the given assumptions on $H(f)$. We note the following, the $\mathrm{CF}, \varphi_{0}(f)$, is the FT of the pdf of the sampling noise, $p_{\tau}(t)$, which means that

$$
\varphi_{0}(f)^{k}=\mathrm{FT}\left[p_{k}(t)\right]=\mathrm{FT}\left[p_{\tau} \star \ldots \star p_{\tau}(t)\right] .
$$

Also, the FT can be moved between the functions in the integral, which, in particular, means that

$$
\left\langle\Delta_{r}, h\right\rangle=\int r(t) h(t) d t=\int \mathrm{FT}[r(t)](f) H(f) d f=\left\langle\Delta_{R}, H\right\rangle .
$$

Now, with this clarified, we can continue:

$$
\begin{aligned}
\left\langle\Delta_{\left.d_{N}^{\mathrm{ARS}}, H\right\rangle}\right. & =\int \sum_{k=0}^{N-1} \varphi_{0}(f)^{k} H(f) d f=\int\left(1+\sum_{k=1}^{N-1} \mathrm{FT}\left[p_{k}(t)\right](f)\right) H(f) d f \\
& =\int H(f) d f+\int \sum_{k=1}^{N-1} \mathrm{FT}\left[p_{k}(t)\right](f) H(f) d f=h(0)+\int g_{N-1}(t) h(t) d t \\
& \rightarrow h(0)+\frac{1}{\mu_{T}} H(0), \quad N \rightarrow \infty .
\end{aligned}
$$

This proves the lemma, since $h(0)=0$ was assumed.

In particular,

$$
\begin{aligned}
\left\langle\Delta_{\left.d_{N}^{\mathrm{ARS}}, \varphi_{0} H\right\rangle}\right. & =\int \sum_{k=1}^{N} \varphi_{0}(f)^{k} H(f) d f=\int \sum_{k=1}^{N} \mathrm{FT}\left[p_{k}(t)\right](f) H(f) d f \\
& =\int g_{N}(t) h(t) d t \rightarrow \frac{1}{\mu_{T}} H(0), \quad N \rightarrow \infty
\end{aligned}
$$

which actually shows that the inverse $\mathrm{FT}$ of $\varphi_{0}(f) H(f)$ is always 0 at the origin, when $H(f)$ fulfills the assumptions in the lemma.

\footnotetext{
${ }^{4}$ The theory for distributions can be revisited in Gausquet and Witkomski (1999) if necessary. For the proof we need to know that $\langle\delta, h\rangle=h(0)$ for $h, \in \mathcal{S}$.
} 
Let us study the conditions on $h(t)$ and $H(f)$ given in Lemma 1 a bit more. The restrictions from the Schwartz class could affect the usability of the lemma. However, all smooth functions with compact support (and their Fourier transforms) are in $\mathcal{S}$, which should suffice for most cases. It is not intuitively clear how hard (32) is. Note that, for any ARS case with continuous sampling noise distribution, $p_{k}(t)$ is approximately a Gaussian for higher $\mathrm{k}$, and we can confirm that, for a large enough fixed $t$,

$$
g_{N}(t)=\sum_{k=1}^{N} \frac{1}{\sqrt{2 \pi k} \sigma_{T}} e^{-\frac{\left(t-k \mu_{T}\right)^{2}}{2 k \sigma_{T}^{2}}} \rightarrow \frac{1}{\mu_{T}}, \quad N \rightarrow \infty .
$$

The integral in (32) can then serve as some kind of mean value approximation, and the edges of $g_{N}(t)$ will not be crucial. Also, condition 3 further restricts the behavior of $h(t)$ for small $t$, which will make condition 2 easier to fulfill. The convergence of $d_{N}^{\mathrm{ARS}}(f)$ is also discussed in Bilinskis and Mikelsons (1992, Ch. 3), for the special case of $g_{\infty}(t)=1 / \mu_{T}$ for all $t$. Discussions on how to achieve this equality is also done.

Now we can study the mean value of $Y(f)$ for ARS, when $N \rightarrow \infty$. Lemma 1 and Theorem 1 give

$$
\begin{aligned}
\mathrm{E}[Y(f)] & =\int_{-\infty}^{\infty} S(\psi) \varphi_{n}(f-\psi) d_{N}^{\mathrm{ARS}}(f-\psi) d \psi=\int S(f+\psi) \varphi_{n}(\psi) d_{N}^{\mathrm{ARS}}(\psi) d \psi \\
& \rightarrow \frac{1}{\mu_{T}} \varphi_{n}(0) S(f)= \begin{cases}S(f) / \mu_{T}, & \text { DirT, } \\
S(f), & \text { RieT. }\end{cases}
\end{aligned}
$$

when $H(\psi)=S(f+\psi) \varphi_{n}(\psi)$ fulfills the requirements in the lemma. For the RieT, condition $3, \int S(f+\psi) \varphi_{1}(\psi) d \psi=0$, is probably the hardest to check, while this condition is automatically fulfilled for DirT, by the special case shown in the proof.

The constant $\mu_{T}$ is the average inter-sampling time and thus the DirT can be scaled in advance to get asymptotically unbiased estimates.

\subsection{Asymptotic Distribution}

The stochastic transform $Y(f)$ given by (24) and (25), is asymptotically a sample from the stochastic distribution

$$
Y(f) \in A s \mathcal{N}\left(\mu_{Y}\left(f ; S, p_{1}^{N}\right), R_{Y}\left(f, f ; S, p_{1}^{N}\right)\right)
$$

where $A s \mathcal{N}$ denotes the asymptotic Gaussian distribution. By this we mean that $Y$ has mean value $\mu_{Y}$ and covariance $R_{Y}$, both depending on $N$, and $Y$ converges in distribution to a Gaussian random variable as $N$ goes to infinity. It is clear that the real and imaginary parts of $Y(f)$ are independent, since cos and sin are orthogonal basis functions.

The transform is a sum of random variables and both $Y(f)$ and the normalized version, $\bar{Y}(f)=\frac{1}{N} Y(f)$, will converge to a Gaussian distribution as $N$ increases. This can be proved by use of a general central limit theorem, and was also indicated by the numerical investigations in Eng and Gustafsson (2005a). In this work, we are satisfied with the fact that the construction of the transform makes the asymptotic Gaussian distribution likely. 


\subsection{Distribution of the Transform Magnitude}

Since the Fourier transform is a complex variable, it will be of importance to study its absolute value. Knowing the distribution of $Y(f)$ to be Gaussian, the asymptotic mean value of the amplitude could be found using the Rice distribution, i.e., (37) implies that

$$
|Y(f)| \in A s \mathcal{R i c},
$$

where $A s \mathcal{R}$ ic denotes the asymptotic Rice distribution. This gives us complete knowledge of the moments of $|Y(f)|$. For the stochastic frequency window $W(f)$, it is therefore possible to calculate the mean value $\mathrm{E}[|W(f)|]$ and covariance $\operatorname{Cov}(|W(f)|)$ numerically, using our expression for $\mu_{W}(f)$ and $R_{W}(f)$ derived earlier and formulas from the Rice distribution.

The Rice distribution is presented in Rice (1945), and below we recapitulate the main ideas. If $z$ is a complex Gaussian stochastic variable with mean $\mu_{z}$, and variance $R_{z}$, then $|z|$ is Rice distributed with known mean $\mu_{|z|}$ and variance $R_{|z|}$. Also, the stochastic variable $r=\left|z-\mu_{z}\right|$ is Rayleigh distributed with known mean $\mu_{r}$ and variance $R_{r}$. These values are related as

$$
\begin{aligned}
\left|\mu_{z}\right| & \leq \mu_{|z|}, \\
\max \left(R_{r}-2 \mu_{r}\left|\mu_{z}\right|, 0\right) & \leq R_{|z|} \leq R_{z} .
\end{aligned}
$$

The moments of the variable $|z|$ can be described, using moments of $z$, as

$$
\mathrm{E}\left[|z|^{k}\right]=R_{z}^{k / 2} e^{-\frac{\left|\mu_{z}\right|^{2}}{R_{z}}} \frac{\Gamma\left(\frac{k+2}{2}\right)}{\Gamma(1)}{ }_{1} F_{1}\left(\frac{k+2}{2}, 1 ; \frac{\left|\mu_{z}\right|^{2}}{R_{z}}\right),
$$

where $\Gamma(q)$ was given in (14). The function ${ }_{1} F_{1}(\alpha, \beta ; x)$ is the confluent hypergeometric function defined as

$$
{ }_{1} F_{1}(\alpha, \beta ; x)=\sum_{n=0}^{\infty} \frac{\Gamma(\alpha+n) \Gamma(\beta) x^{n}}{\Gamma(\alpha) \Gamma(\beta+n) n !} .
$$

For even values of $k$ it is possible to get polynomial expressions for $\mathrm{E}\left[|z|^{k}\right]$, for example,

$$
\begin{aligned}
& \mathrm{E}\left[|z|^{2}\right]=\left|\mu_{z}\right|^{2}+R_{z}, \\
& \mathrm{E}\left[|z|^{4}\right]=\left|\mu_{z}\right|^{4}+4\left|\mu_{z}\right|^{2} R_{z}+2 R_{z}^{2} .
\end{aligned}
$$

\subsection{The Periodogram}

The asymptotic distribution of $Y(f)$ will enforce the asymptotic distribution on the periodogram, $P_{Y}(f)$. We note that known connection between the Gaussian, Rice, Rayleigh and exponential distributions gives the following statements for a complex random variable $z$,

$$
z \in \mathcal{N}(\mu, R) \Rightarrow\left\{\begin{aligned}
|z| & \in \mathcal{R i c} \\
|z-\mu| & \in \mathcal{R} \text { ay }(R / 2) \\
|z-\mu|^{2} & \in \mathcal{E x p}\left(\frac{1}{R}\right)
\end{aligned}\right.
$$


This means that (37), together with the fact that the real and imaginary parts of $Y(f)$ are independent, implies

$$
\begin{aligned}
\left|Y(f)-\mu_{Y}(f)\right|^{2} & \in A s \mathcal{E} \operatorname{xp}\left(\frac{1}{R_{Y}(f)}\right), \\
\left|Y(f)-\mu_{Y}(f)\right|^{2} & =P_{Y}(f)+\left|\mu_{Y}(f)\right|^{2}-X(f) \\
X(f) & =2 \Re\left(Y(f) \mu_{Y}(f)^{*}\right) \\
& \in A s \mathcal{N}\left(2\left|\mu_{Y}(f)\right|^{2}, 2\left|\mu_{Y}(f)\right|^{2} R_{Y}(f, f)\right)
\end{aligned}
$$

where $A s \mathcal{E}$ xp denotes the asymptotic exponential distribution and $\Re$ is the real value. The asymptotic distribution for the periodogram, $P_{Y}(f)$, is shown in (41) to be constructed by a sum of one Gaussian and one exponential random variable, i.e., we can write

$$
\begin{aligned}
& P_{Y}(f)=\left|\mu_{Y}(f)\right|^{2}+X_{1}(f)+X_{2}(f), \\
& X_{1}(f) \in A s \mathcal{E} \operatorname{xp}\left(\frac{1}{R_{Y}(f)}\right) \\
& X_{2}(f) \in A s \mathcal{N}\left(0,2\left|\mu_{Y}(f)\right|^{2} R_{Y}(f, f)\right) .
\end{aligned}
$$

We can also use the Rice distribution to find the asymptotic variance of $P_{Y}(f)$. From (40) we get

$$
\begin{aligned}
R_{P}(f) & =\operatorname{Var}\left(P_{Y}(f)\right)=E\left[|Y(f)|^{4}\right]-\mu_{P}(f)^{2}, \\
& =\left|\mu_{Y}(f)\right|^{4}+4\left|\mu_{Y}(f)\right|^{2} R_{Y}(f, f)+2 R_{Y}(f, f)^{2}-\left(\left|\mu_{Y}(f)\right|^{2}+R_{Y}(f, f)\right)^{2}, \\
& =2\left|\mu_{Y}(f)\right|^{2} R_{Y}(f, f)+R_{Y}(f, f)^{2}=R_{Y}(f, f)\left(2 \mu_{P}(f)-R_{Y}(f, f)\right),
\end{aligned}
$$

where $\mu_{P}(f)$ is given by Corollary 1 and $R_{Y}(f, f)$ are given by Theorem 1 . This is in perfect correspondance with equation $(42)\left(R_{P}=\operatorname{Var}\left(X_{1}\right)+\operatorname{Var}\left(X_{2}\right)\right)$ and actually shows that $X_{1}(f)$ and $X_{2}(f)$ are independent, which was not intuitively clear.

We can keep in mind that the usual statistical calculations for the periodogram, for example, by Scargle (1982) and Stoica and Moses (2005), are done based on stochastic signals and not stochastic sampling instants. Thus, this defines the a priori distribution (or mean and variance) of the Lomb-Scargle periodogram apart from the scaling factor.

\section{Examples and Discussion}

This section provides illustrations of the frequency window and the periodogram, without inclusion of the amplitude noise, to further demonstrate the effect of sampling noise. Also, calculations in the case of both sampling and amplitude noise are given.

\subsection{The Stochastic Frequency Window}

In Figure 1, the mean values of $\frac{1}{N} W(f)$ are shown ${ }^{5}$ together with one realization, for the case of ARS and SJS, respectively. The result is given for the DirT. The

\footnotetext{
${ }^{5}$ The scaling with $N$ is done to make different plots comparable in the paper, since $\mathrm{E}[W(0)]=N$.
} 
figures also include a confidence band of one standard deviation given by the covariance description in (28b). Note that, since $W(f)$ is complex, $|W(f)|$ is plotted together with $\left|\mu_{W}\right|$ and $\left|\mu_{W}+\sqrt{R_{W}}\right|$.

For the DirT, $W(0)$ is a constant and hence, the variance is zero, which is confirmed by the figures. This means that for the DirT, the sampling noise only affects the side lobes and the width of the main lobe of the stochastic frequency window. In the case of SJS, the leakage is the standard leakage with amplitude scaling since $\mu_{W}(f)=\varphi_{0}(f) d_{N}^{\mathrm{SJS}}(f)$, and the periodic $d_{N}^{\mathrm{SJS}}(f)$ is what we get when no sampling noise is present, cf., (30). We also note that significant leakage effects can be expected for a given realization of $\left\{t_{k}\right\}$.

\subsection{The Periodogram}

The effect of stochastic sampling is more conveniently illustrated on the realvalued periodogram. We restrict the investigation to a single frequency and get Figure 2, which shows one realization,

$$
P_{W}(f)=|W(f)|^{2}
$$

together with the first two moments,

$$
\begin{aligned}
& \mu_{P}(f)=\left|\mu_{W}(f)\right|^{2}+R_{W}(f, f), \\
& R_{P}(f)=\mu_{P}(f)+\sqrt{R_{W}(f, f)\left(2\left|\mu_{W}(f)\right|^{2}+R_{W}(f, f)\right)},
\end{aligned}
$$

as an example of the periodogram. The setup is identical to the one for Figure 1a, i.e., ARS and DirT is used. It is clear that the leakage is reduced for a single realization compared to the visualization of the transform in the previous section. However, the mean value of the tails of the periodogram never goes to zero, so there is permanent leakage due to the stochastic sampling. The spurious peaks looks like aliasing, and will also depend on the particular realization.

We also include the result for a multi-sine,

$$
s(t)=a_{1} \sin \left(2 \pi f_{1} t\right)+a_{2} \sin \left(2 \pi f_{2} t\right),
$$

to show the cross effects between frequencies. In this example we chose $a_{1}=1.2$, $a_{2}=0.8, f_{1}=1$ and $f_{2}=2$. We study both the AFT and the periodogram in Figure 3 , and find that the mean value is informative, while the single realization contains spurious peaks and a large noise level. This is particularly true for the AFT.

To conclude this investigation, we give some advice on appropriate actions when sampling noise is present.

- When the sampling noise distribution is known, it is easy to calculate mean and variance of the periodogram to get an idea of the achiveable performance. Background noise will always be present.

- For specific realizations, smoothing of the periodogram can probably improve performance. 


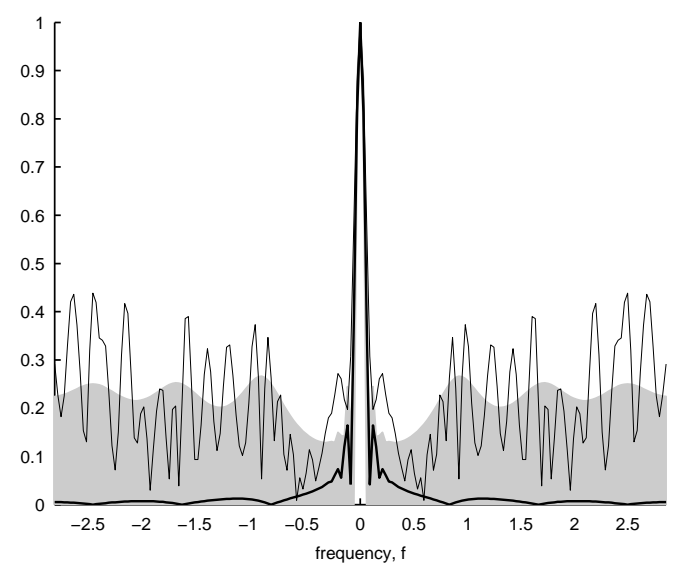

(a) ARS with rectangular sampling noise between 0.1 and 1.3

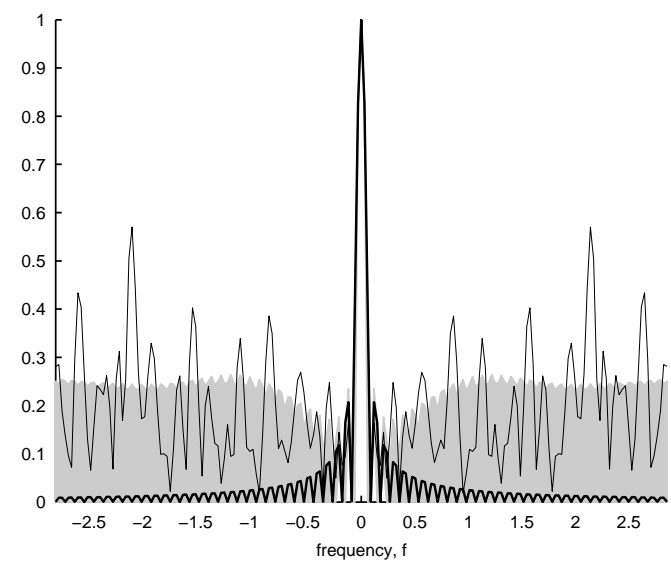

(b) SJS with rectangular sampling noise between -0.35 and 0.35

Figure 1: Mean value $\left|\mu_{W}(f)\right|$ (thick line), confidence band $\left|\mu_{W} \pm \sqrt{R_{W}}\right|$ (gray shade) and one realization $W(f)$ (thin line) of the stochastic window for the DirT, for two different sampling noise distributions, with $N=16$ and $T=0.7$. 


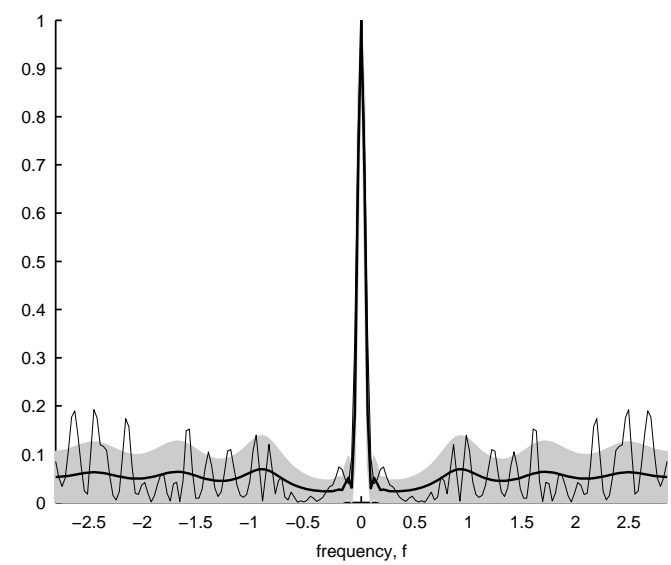

Figure 2: Mean value $\mu_{P}(f)$ (thick line), confidence band $\mu_{P} \pm \sqrt{R_{P}}$ (gray shade) and one realization $P_{W}(f)$ (thin line) of the periodogram $|W(f)|^{2}$, when ARS and DirT is used.

\subsection{Addition of Amplitude Noise}

The common case, where the signal is corrupted by amplitude noise is easily included in this setup. Let

$$
y_{k}=s\left(t_{k}\right)+v_{k},
$$

where $v_{k}$ is a sequence of white Gaussian noise with variance $\sigma_{v}^{2}$, also independent of $s\left(t_{k}\right)$. The AFT is then extended with the term $V(f)$ as in

$$
Y^{e}(f)=\sum_{k} s\left(t_{k}\right) e^{-i 2 \pi f t_{k}}+\sum_{k} v_{k} e^{-i 2 \pi f t_{k}}=Y(f)+V(f) .
$$

Superscript $e$ stands for the extended AFT, here exemplified with the DirT. Since $t_{k}$ and $v_{k}$ are independent we simply get

$$
\begin{aligned}
\mathrm{E}\left[Y^{e}(f)\right] & =\mu_{Y}(f)+0, \\
\operatorname{Var}\left(Y^{e}(f)\right) & =R_{Y}(f, f)+N \sigma_{v}^{2},
\end{aligned}
$$

for the extended mean value and variance. The additional term $V(f)$ is also Gaussian distributed, and hence the extended AFT is still asymptotically Gaussian. Therefore the discussion for the periodogram holds without change. In case with only amplitude noise, $y_{k}=s(k T)+v_{k}$, we recall that the periodogram is exponentially distributed, which is given directly from the Gaussian distribution of the noise term. In the previous section, we saw the results for sampling noise. Now, the extended periodogram is

$$
\left|Y^{e}(f)\right|^{2}=|Y(f)+V(f)|^{2}=|Y(f)|^{2}+|V(f)|^{2}+2 \Re(Y(f) V(f))
$$

and we have all knowledge to describe mean, variance and distribution. The distribution is once again a sum of an exponential and a Gaussian random variable. The first two moments are

$$
\begin{aligned}
\mathrm{E}\left[\left|Y^{e}(f)\right|^{2}\right] & =\left|\mu_{Y}(f)\right|^{2}+R_{Y}(f, f)+N \sigma_{v}^{2}, \\
\operatorname{Var}\left(\left|Y^{e}(f)\right|^{2}\right) & =2\left|\mu_{Y}(f)\right|^{2} R_{Y}(f, f,)+R_{Y}(f, f)^{2}+\left(N \sigma_{v}^{2}\right)^{2}
\end{aligned}
$$




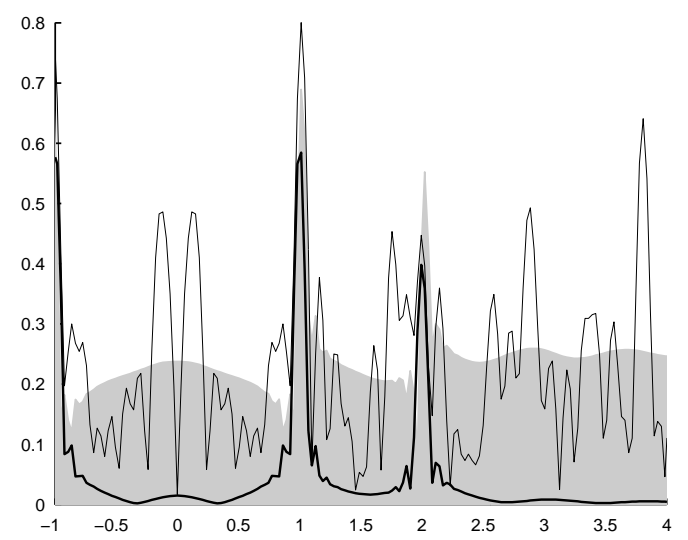

(a) The AFT

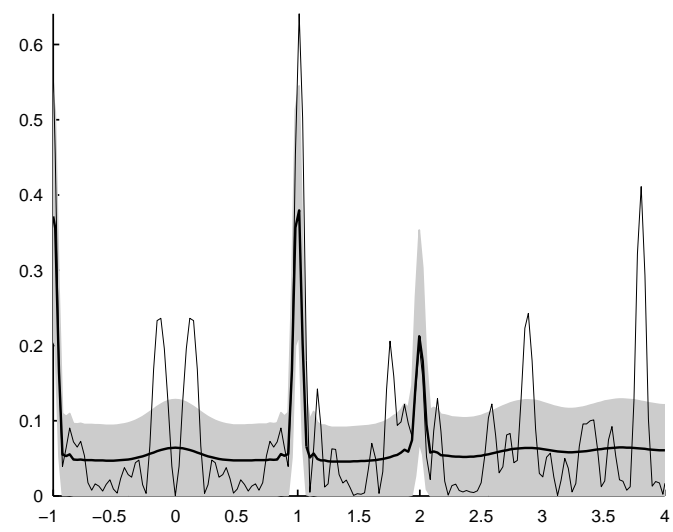

(b) The periodogram

Figure 3: Mean value (thick line), confidence band (gray shade) and one realization (thin line) of the AFT and periodogram, when the signal is a multi-sine, (43), the DirT is used, and the sampling ARS setup is identical to the one in Figure 1a. 
After some effort, this is given by previous calculations on the Rice distribution and the periodogram without amplitude noise in Corollary 1 and Section 5.4, noting only that $Y(f)$ and $V(f)$ are independent.

\section{Conclusions}

Two types of linear frequency transforms have been investigated for signals with non-uniform sampling times. The stochastic properties of the transforms have been studied for both additive random sampling and stochastic jitter sampling. The investigations resulted primarily in analytical expressions for bias and covariance, and an asymptotic expression for the mean value. These results were applied to periodogram computations, where both non-asymptotic and asymptotic expressions were given. It was also shown that regular amplitude noise is easily can be included in the description.

\section{Acknowledgment}

The authors thank Ph.D. Martin Enqvist for sharing ideas and for many interesting discussions.

\section{Appendices}

Here we provide the parts of the proof for Table 4, Theorem 2, that was left out in the presentation. We use the following separation for the covariance calculation

$$
\begin{aligned}
\mathrm{E}\left[W(f) W^{*}(\psi)\right] & =\sum_{k=1}^{N} \sum_{l=1}^{N} \mathrm{E}\left[\left(T_{k} T_{l}\right)^{n} e^{-i 2 \pi f t_{k}} e^{i 2 \pi \psi t_{l}}\right] \\
& =\sum_{k=1}^{N} \sum_{l=1}^{k-1} \cdots+\sum_{k=1}^{N} \sum_{l=k}^{k} \cdots+\sum_{k=1}^{N} \sum_{l=k+1}^{N} \cdots \\
& =\Sigma_{1}(f, \psi)+\Sigma_{2}(f, \psi)+\Sigma_{3}(f, \psi) .
\end{aligned}
$$

Each term is treated separately for convenience and the expected value is taken under the assumption of independent sampling noise, $\tau_{k}$. The notation was given in Table 3.

\section{A Covariance of $W(f)$ Using ARS}

Note that $T_{k}=\tau_{k}$ for ARS, and the sum over $l<k$ evaluates to

$$
\begin{aligned}
\Sigma_{1}(f, \psi) & =\sum_{k=1}^{N} \sum_{l=1}^{k-1} \mathrm{E}\left[\left(\tau_{k} \tau_{l}\right)^{n} \prod_{j=l+1}^{k} e^{-i 2 \pi f \tau_{j}} \prod_{m=1}^{l} e^{-i 2 \pi(f-\psi) \tau_{m}}\right] \\
& =\sum_{k=1}^{N} \sum_{l=1}^{k-1} \varphi_{n}(f) \varphi_{0}(f)^{k-1-l} \varphi_{n}(f-\psi) \varphi_{0}(f-\psi)^{l-1}
\end{aligned}
$$


The second term is the sum when $k$ and $l$ are equal,

$$
\begin{aligned}
\Sigma_{2}(f, \psi) & =\sum_{k=1}^{N} \mathrm{E}\left[\tau_{k}^{2 n} \prod_{j=1}^{k} e^{-i 2 \pi(f-\psi) \tau_{j}}\right]=\sum_{k=1}^{N} \varphi_{2 n}(f-\psi) \varphi_{0}(f-\psi)^{k-1} \\
& =\varphi_{2 n}(f-\psi) d_{N}^{\mathrm{ARS}}(f-\psi),
\end{aligned}
$$

and the last term is given by symmetry,

$$
\begin{aligned}
\Sigma_{3}(f, \psi) & =\sum_{k=1}^{N} \sum_{l=k+1}^{N} \mathrm{E}\left[\left(\tau_{k} \tau_{l}\right)^{n} \prod_{j=k+1}^{l} e^{i 2 \pi \psi \tau_{j}} \prod_{m=1}^{k} e^{i 2 \pi(f-\psi) \tau_{m}}\right] \\
& =\sum_{l=1}^{N} \sum_{k=1}^{l-1} \mathrm{E}\left[\left(\tau_{k} \tau_{l}\right)^{n} \prod_{j=k+1}^{l} e^{i 2 \pi \psi \tau_{j}} \prod_{m=1}^{k} e^{i 2 \pi(f-\psi) \tau_{m}}\right]=\Sigma_{1}(-\psi,-f) .
\end{aligned}
$$

Identification of terms proves the expression in (28b) for ARS.

\section{B Covariance of $W(f)$ Using SJS}

The calculations are very similar to those in the previous section, and we skip a few of the intermediate steps. Note that $T_{k}$ and $T_{l}$ are independent only when $|k-l|>1$, which will affect the expressions when $n=1$. This requires that we also consider the sums over $k=l+1$ and $k=l-1$ as special cases.

First, we consider the three simplest cases

$$
\begin{aligned}
\Sigma_{1}(f, \psi) & =\sum_{k=1}^{N} \sum_{l=1}^{k-2} \mathrm{E}\left[\left(T_{k} T_{l}\right)^{n} e^{-i 2 \pi f \tau_{k}} e^{i 2 \pi \psi \tau_{l}} e^{-i 2 \pi f k T} e^{i 2 \pi \psi l T}\right] \\
& =\sum_{k=1}^{N} \sum_{l=1}^{k-2} \varphi_{n}(f) \varphi_{n}(-\psi) e^{-i 2 \pi(f k-\psi l) T} \\
\Sigma_{2}(f, \psi) & =\sum_{k=1}^{N} \mathrm{E}\left[\left(T_{k}\right)^{2 n} e^{-i 2 \pi(f-\psi) \tau_{k}} e^{-i 2 \pi(f-\psi) k T}\right] \\
& =\varphi_{2 n}(f-\psi) d_{N}^{\mathrm{SJS}}(f-\psi), \\
\Sigma_{3}(f, \psi) & =\sum_{k=1}^{N} \sum_{l=k+2}^{N} \mathrm{E}\left[\left(T_{k} T_{l}\right)^{n} e^{i 2 \pi \psi\left(l T+\tau_{l}\right)} e^{-i 2 \pi f\left(k T+\tau_{k}\right)}\right] \\
& =\varphi_{n}(f) \varphi_{n}(-\psi) \sum_{l=1}^{N} \sum_{k=1}^{l-2} e^{-i 2 \pi(f k-\psi l) T}=\Sigma_{1}(-\psi,-f) .
\end{aligned}
$$

Now, when $k=l-1$,

$$
\begin{aligned}
\Sigma_{1 x}(f, \psi) & =\sum_{k=1}^{N} \mathrm{E}\left[\left(T_{k} T_{k+1}\right)^{n} e^{-i 2 \pi f\left(k T+\tau_{k}\right)} e^{i 2 \pi \psi\left(k T+T+\tau_{k+1}\right)}\right], \\
& =\mathrm{E}\left[\left(T_{k} T_{k+1}\right)^{n} e^{-i 2 \pi f \tau_{k}} e^{i 2 \pi \psi \tau_{k+1}}\right] \sum_{k=1}^{N} e^{-i 2 \pi(f-\psi) k T} e^{i 2 \pi \psi T},
\end{aligned}
$$


and, when $k=l+1$,

$$
\begin{aligned}
\Sigma_{3 x}(f, \psi) & =\sum_{l=1}^{N} \mathrm{E}\left[\left(T_{l+1} T_{l}\right)^{n} e^{-i 2 \pi f\left((l+1) T+\tau_{l+1}\right)} e^{i 2 \pi \psi\left(l T+\tau_{l}\right)}\right], \\
& =\mathrm{E}\left[\left(T_{l} T_{l+1}\right)^{n} e^{-i 2 \pi f \tau_{l+1}} e^{i 2 \pi \psi \tau_{l}}\right] \sum_{l=1}^{N} e^{-i 2 \pi(f-\psi) l T} e^{-i 2 \pi f T} .
\end{aligned}
$$

From this (29g) can be extracted, and it remains to evaluate the expected value, outside the sum, in the two latest expressions. When $n=0$,

$\mathrm{E}\left[\left(T_{k} T_{k+1}\right)^{n} e^{-i 2 \pi f \tau_{k}} e^{i 2 \pi \psi \tau_{k+1}}\right]=\mathrm{E}\left[\left(T_{l} T_{l+1}\right)^{n} e^{-i 2 \pi f \tau_{l+1}} e^{i 2 \pi \psi \tau_{l}}\right]=\varphi_{0}(f) \varphi_{0}(-\psi)$.

For $n=1$ the calculations are more involved, and it will be helpful to note the following connections

$$
\begin{aligned}
\mathrm{E}\left[\left(T+\tau_{k}\right) e^{-i 2 \pi f \tau_{k}}\right] & =\varphi_{1}(f), \\
\mathrm{E}\left[\tau_{k} e^{-i 2 \pi f \tau_{k}}\right] & =\varphi_{1}(f)-T \varphi_{0}(f), \\
\mathrm{E}\left[\tau_{k}^{2} e^{-i 2 \pi f \tau_{k}}\right] & =\varphi_{2}(f)-T \varphi_{1}(f)-\mathrm{E}\left[\tau_{k-1}^{2}\right] \varphi_{0}(f),
\end{aligned}
$$

which are given by the derivation from the definition of $\varphi_{m}(f)=\mathrm{E}\left[\left(T_{k}\right)^{m} e^{-i 2 \pi f \tau_{k}}\right]$. Since $t_{k}=T+\tau_{k}-\tau_{k-1}$ for SJS, we get, with the equations above,

$$
\begin{aligned}
T_{k} T_{k+1}=\left(T+\tau_{k}-\tau_{k-1}\right)\left(T+\tau_{k+1}-\tau_{k}\right) & \\
=\left(T^{2}-\right. & \left.\tau_{k}^{2}\right)+\left(T+\tau_{k}\right) \tau_{k+1}-\tau_{k-1}\left(T-\tau_{k}\right)-\tau_{k}^{2} \\
\mathrm{E}\left[T_{k} T_{k+1} e^{-i 2 \pi f \tau_{l+1}} e^{i 2 \pi \psi \tau_{l}}\right]= & \left(T^{2} \varphi_{0}(f)-\mathrm{E}\left[\tau_{k}^{2} e^{-i 2 \pi f \tau_{k}}\right]\right) \varphi_{0}(-\psi) \\
& +\varphi_{1}(f) \mathrm{E}\left[\tau_{k+1} e^{i 2 \pi \psi \tau_{k+1}}\right]-0-\mathrm{E}\left[\tau_{k}^{2} e^{-i 2 \pi f \tau_{k}}\right] \varphi_{0}(-\psi) \\
= & \kappa(f) \varphi_{0}(-\psi)+\varphi_{1}(f) \varphi_{1}(-\psi)
\end{aligned}
$$

This was the only part left in (29d) to show, and (28b) for SJS is now proved.

\section{References}

Benedetto, John J (1992). Irregular sampling and frames. In Chui, Charles K (Ed.), Wavelets: A Tutorial in Theory and Practice. Academic Press Inc.

Beurling, Arne (1989). Interpolation for an interval on $R^{1}$. In Collected Works of Arne Beurling, vol. 2. Birkhäuser Verlag.

Beutler, Frederick J (1970). Alias-free randomly timed sampling of stochastic processes. In IEEE Trans. Inform. Theory, vol. 16(2):pp. 147-152. ISSN 0018-9448.

Bilinskis, Ivar and Mikelsons, Arnolds (1992). Randomized Signal Processing. Prentice Hall.

Bland, Denise M; Laakso, Timo I; and Tarczynski, Andrzej (1996). Analysis of algorithms for nonuniform-time discrete Fourier transform. In IEEE Int. Symp. on Circuits and Syst. Atlanta, Georgia, USA. 
Bland, Denise M and Tarczynski, Andrzej (1997). Optimum nonuniform sampling sequence for alias frequency suppression. In IEEE Int. Symp. on Circuits and Syst. Hong Kong.

Eng, Frida and Gustafsson, Fredrik (2005a). Frequency transforms based on nonuniform sampling — basic stochastic properties. In $R V K$. Linköping, Sweden.

Eng, Frida and Gustafsson, Fredrik (2005b). System identification using measurements subject to stochastic time jitter. In IFAC World Congress. Prague, Czech Republic.

Feichtinger, Hans G and Gröchenig, Karlheinz (1994). Theory and practice of irregular sampling. In Benedetto, John J and Frazier, Michael W (Eds.), Wavelets: Mathematics and Applications. CRC Press.

Gausquet, Claude and Witkomski, Patrick (1999). Fourier Analysis and Applications. Springer Verlag.

Gunnarsson, Frida; Gustafsson, Fredrik; and Gunnarsson, Fredrik (2004). Frequency analysis using nonuniform sampling with applications to adaptive queue management. In IEEE Int. Conf. on Acoust., Speech, Signal Processing. Montreal, Canada.

Hall, Peter and Yin, Yiying (2004). Nonuniform sequential sampling for signal analysis. In IEEE Trans. Inform. Theory, vol. 50(9):pp. 2081-2088. ISSN 0018-9448.

Ljung, Lennart (1999). System Identification - Theory for the User. Prentice Hall, 2 ed.

Lomb, N R (1976). Least-squares frequency analysis of unequally spaced data. In Astrophysics and Space Science, vol. 39:pp. 447-462.

Marvasti, Farokh (Ed.) (2001). Nonuniform Sampling: Theory and Practice. Kluwer Academic Publishers.

Masry, Elias (1978). Alias-free sampling: An alternative conceptualization and its applications. In IEEE Trans. Inform. Theory, vol. 24(3):pp. 317-324.

Masry, Elias (1983). Probability density estimation from sampled data. In IEEE Trans. Inform. Theory, vol. 29(5):pp. 696-709.

Papenfuss, Frank; Artyukh, Yuri; Boole, E; and Timmermann, Dirk (2003). Optimal sampling functions in nonuniform sampling driver designs to overcome the Nyquist limit. In IEEE Int. Conf. on Acoust., Speech, Signal Processing. Hong Kong.

Persson, Niclas (2002). Event Based Sampling with Application to Spectral Estimation. Licentiate Thesis No. 981, Dep. of Electr. Eng., Linköpings universitet, Sweden.

URL http://www.control.isy.liu.se/

Rice, Stephen O (1945). Mathematical analysis of random noise, Part III. In The Bell System Technical Journal, vol. 24:pp. 46-156. 
Scargle, Jeffrey D (1982). Studies in astronomical time series analysis II: Statistical aspects of spectral analysis of unevenly spaced data. In The Astrophysical Journal, vol. 263:pp. 835-853.

Schoukens, Johan; Pintelon, Rik; and Hamme, Hugo Van (1992). The interpolated fast Fourier transform: a comparative study. In IEEE Trans. Instrum. Meas., vol. 41(2):pp. 226-232. ISSN 0018-9456.

Shapiro, Harold S and Silverman, Richard A (1960). Alias-free sampling of random noise. In Journal of the Society for Industrial and Applied Mathematics, vol. $8(2)$ :pp. $225-248$.

van Steenis, H. G. and Tulen, J. H. M. (1991). A comparison of two methods to calculate the heart rate spectrum based on non-equidistant sampling. In Int. Conf. of the IEEE Eng. in Med. and Biol. Soc. Philadelphia, Pennsylvania, USA.

Stoica, Petre and Moses, Randolph L (2005). Spectral Analysis of Signals. Prentice Hall. ISBN 0-13-113956-8.

Tarczynski, Andrzej and Allay, Najib (2004). Spectral analysis of randomly sampled signals: Suppression of aliasing and sampler jitter. In IEEE Trans. Signal Processing, vol. 52(12):pp. 3324-3334. ISSN 1053-587X.

Wojtkiewicz, Andrzej and Tuszyński, Michal (1992). Application of the Dirichlet transform in analysis of nonuniformly sampled signals. In IEEE Int. Conf. on Acoust., Speech, Signal Processing. San Fransisco, California, USA. 